\title{
DESENVOLVIMENTO FONOLÓGICO EM CRIANÇAS DOS 3 ANOS E 6 MESES AOS 4 ANOS E 6 MESES DE IDADE NASCIDAS COM MUITO BAIXO PESO
}

\author{
Patrícia Machado NOGUEIRA* \\ Maria João FREITAS ${ }^{* *}$
}

- RESUMO: O presente trabalho tem o objectivo central de avaliar o desenvolvimento fonológico de crianças nascidas com muito baixo peso, contribuindo com evidência empírica para a reflexão sobre o papel das variáveis fonológicas na avaliação do desenvolvimento linguístico infantil. Foi implementado um estudo com 30 crianças na faixa etária dos 3 anos e 6 meses aos 4 anos e 6 meses, sendo que 15 pertenciam à população de crianças nascidas com muito baixo peso e as restantes à de crianças nascidas com peso adequado, emparelhadas por género e idade. Observou-se o desempenho segmental das crianças nos dois grupos em estudo, avaliando-se a relação entre a produção das consoantes do inventário segmental do Português Europeu e a sua distribuição em função das variáveis fonológicas posição na palavra, posição na sílaba e acento de palavra. O objectivo central foi o de testar a eficácia das variáveis fonológicas acima referidas na identificação de diferenças de desenvolvimento fonológico entre os dois grupos de crianças. Através do teste de QuiQuadrado e da comparação frequencial, verificou-se que as crianças nascidas com muito baixo peso apresentavam um desempenho verbal inferior ao das crianças com peso adequado, no que diz respeito às variáveis fonológicas testadas.

- PALAVRAS-CHAVE: Crianças de muito baixo peso. Desenvolvimento fonológico. Palavra. Sílaba. Constituintes silábicos. Acento de palavra.

\section{ASPECTOS FONOLÓGICOS}

Vários trabalhos sobre o desenvolvimento linguístico infantil têm identificado e/ou testado, nas últimas três décadas, as variáveis linguísticas com impacto na aquisição de diferentes sistemas gramaticais, tanto em contexto monolingue como bilingue. Este trabalho tem sido determinante para a definição da forma como o conhecimento linguístico se estrutura no processo de desenvolvimento cognitivo infantil, contribuindo, assim, para a validação de modelos teóricos

\footnotetext{
* UALG - Universidade do Algarve. Escola Superior de Saúde. Instituto de Ciências da Saúde. Faro - Portugal. 8000-510 - patricianogueira2@gmail.com

** UL- Universidade de Lisboa. Faculdade de Letras. Centro de Linguística. Lisboa - Portugal. 1600-214 joaofreitas@fl.ul.pt
} 
de representação deste tipo de conhecimento (INGRAM, 1989; FLETCHER; MCWHINNEY, 1995; GUASTI, 2002; BALL, et al., 2008; BAVIN, 2009).

A componente fonológica da gramática integra o processamento das unidades de natureza segmental mas também as de natureza prosódica, como a sílaba, o acento (pé), a palavra e os sintagmas (fonológico e entoacional), que permitem descrever a estrutura prosódica dos enunciados de fala (NESPOR; VOGEL, 1986; MATEUS; ANDRADE, 2000; BISOL, 2005). O foco específico em alguns destes constituintes fonológicos - o segmento, a sílaba e a palavra, unidades estudadas no presente artigo - tem permitido testar a sua eficácia na avaliação das produções das crianças, sendo estas unidades apontadas como bons indicadores do desenvolvimento fonológico infantil (BERNHARDT; STEMBERGER, 1998; CORREIA, 2004; COSTA, et al., 2007; FREITAS, 1997; FREITAS, et al., 2006; OLIVEIRA, et al., 2004; SMITH, 2004; VIHMAN, 1996; YAVAS; HERNANDORENA; LAMPRECHT, 2002).

No âmbito da fonologia multilinear, assume-se uma existência de relações de interdependência entre os vários constituintes fonológicos, representadas sob a forma de estruturas arbóreas, que implicam o conceito de constituência interna (CLEMENTS, 1985; NESPOR; VOGEL, 1986; MCCARTHY; PRINCE, 1986; GOLDSMITH, 1995; MATEUS; ANDRADE, 2000; BISOL, 2005). Vários estudos têm demonstrado a adequação dos modelos fonológicos propostos no âmbito da fonologia multilinear à descrição e à interpretação de dados da produção de crianças com desenvolvimento típico (FIKKERT, 1994, 2005, 2007; ARCHIBALD, 1995; MACKEN, 1995; MENN; STOEL-GAMMON, 1995; FREITAS, 1997; BERNHARDT; STEMBERGER, 1998; LAMPRECHT, 1999; ROSE, 2000; SANTOS, 2001; MATZENAUER, 2001; LAMPRECHT, et al., 2004; KAGER; PATER; ZONNEVELD, 2004; AVERY; DRESHER, 2008; DEMUTH, 2009; COSTA, 2010; JOHNSON; REIMERS, 2010; ALMEIDA, 2011). Estes mesmos instrumentos de análise têm também sido usados por vários investigadores e profissionais nos domínios da avaliação e da intervenção clínicas de crianças com desenvolvimento atípico, com efeitos testados na redução do tempo e na eficácia da intervenção terapêutica (MATZENAUER, 1988; YAVAS; HERNANDORENA; LAMPRECHT, 1991; BERNHARDT; STEMBERGER, 2000; BISHOP; LEONARD, 2000; MOTA, 2001; KESKE-SOARES, 2001; FAVA, 2002; LAMPRECHT, et al., 2004; DUARTE, 2006; DINNSEN; GIERUT, 2008; INGRAM, 2008; LAZZAROTTO-VOLCÃO, 2009).

A presença de relações hierárquicas entre os vários constituintes fonológicos, espoletou, durante os anos 90, a discussão sobre a natureza bottom-up ou topdown do processamento inerente ao estabelecimento destas relações, tanto na representação do conhecimento fonológico adulto como na representação da sua estruturação gradual durante os primeiros anos de vida. Dada a natureza holística do processo de desenvolvimento linguístico, diversos estudos avaliaram 
as potenciais relações entre os vários constituintes que integram o conhecimento fonológico. Muitos dos trabalhos em aquisição da fonologia mostraram que domínios prosódicos hierarquicamente mais altos estabelecem fortes restrições sobre a aquisição de unidades de domínios mais baixos (FIKKERT, 2007; BERNHARDT; STEMBERGER, 1998), apresentando resultados relativos ao efeito de variáveis prosódicas como posição na palavra (inicial, medial, final), posição na sílaba (Ataque, Rima, Núcleo, Coda) e acento de palavra (padrões proparoxítono, paroxítono e oxítono) na aquisição do inventário segmental de uma língua (FIKKERT, 1994; FREITAS, 1997; LLEÓ; PRINZ, 1997; MATZENAUER, 2001; LAMPRECHT, et al., 2004; KIRK; DEMUTH, 2003). Por outras palavras, a literatura especializada tem demonstrado a existência de fortes relações entre a emergência e estabilização de uma dada unidade segmental e as suas propriedades prosódicas, decorrentes dos constituintes prosódicos superiores que as hospedam na língua-alvo, informação crucial para uma avaliação adequada do desenvolvimento fonológico infantil, tanto típico como atípico. A título ilustrativo, em línguas como o Português Europeu (PE), a aquisição de um segmento como o /1/, cuja produção é possível em três posições silábicas (em Ataque simples (leite), como $\mathrm{C}_{2}$ de um Ataque ramificado (fllor) e em Coda (balde)), não é categórica mas sim gradual: o segmento estabiliza primeiro em Ataque simples, sendo o Ataque ramificado a última estrutura a exibir a sua estabilização (FREITAS, 1997). Ora, a presença do segmento numa única posição prosódica, num dado instrumento de avaliação da linguagem, não permitirá uma avaliação rigorosa do desenvolvimento fonológico da criança em foco. A ausência de produção desta lateral pode decorrer não da destreza da criança para articular o som ou da sua não aquisição como entidade do inventário fonológico da língua, mas da disponibilização de um dado constituinte prosódico. Se a criança já produzir adequadamente o segmento em palavras como "leite" mas não em palavras como "balde" e "flor", tal decorrerá da não disponibilização dos constituintes silábicos Coda e Ataque Ramificado e não da ausência da lateral alveolar no sistema fonológico da criança. Nestes casos, a intervenção terapêutica deve focar-se no trabalho sobre a relação entre os constituintes silábicos e o segmento /1/ e não, exclusivamente, no trabalho sobre o segmento /1/.

Ainda na sequência da natureza holística do processo de desenvolvimento linguístico, a investigação tem explorado o efeito das interfaces gramaticais na aquisição de determinadas línguas (MORGAN; DEMUTH, 1996; ABAURRE; SCARPA; GALVES, 1999; SCARPA, 1999; WEISSENBORN; HOHLE, 2001). No caso do PE, verificou-se que a emergência e estabilização das Codas é gradual: ocorre em primeiro lugar em Coda final, tendencialmente átona, dada a frequência elevada de formas paroxítonas no PE (patoss); a sua aquisição em Coda tónica final é também precoce (maçãs, nariz ) e precede a da Coda tónica medial (feșta); a última estrutura a ser produzida corresponde à da Coda átona medial (vestido). 
Esta ordem viola a esperada estabilização inicial das estruturas tónicas (FIKKERT, 1994). A aquisição precoce das Codas átonas finais (patosi) no PE, imune ao efeito da proeminência acústica das estruturas tónicas, tem sido interpretada como o produto da interface fonologia-morfologia, colocando-se a hipótese de esta Coda em final de palavra ser processada pelas crianças portuguesas como proeminente por lhe ser inerente informação de dois tipos: fonológica (ramificação da Rima, estrutura não disponível no início da aquisição) e morfológica (marcação do número plural; o final de palavra em PE está associado à presença de informação flexional em diferentes classes de palavras, o que a torna gramaticalmente proeminente). A confluência de informação de dois módulos gramaticais numa só estrutura estaria, assim, na base da sua aquisição precoce (FREITAS; MIGUEL; FARIA, 2001).

\section{ASPECTOS CLÍNICOS}

Nas últimas décadas, o crescimento científico associado aos avanços tecnológicos na área perinatal, ao desenvolvimento do conhecimento sobre a fisiopatologia de vários problemas associados à prematuridade e à expansão dos recursos de diagnóstico e terapêuticas permitiu um aumento significativo da sobrevivência de recém-nascidos pré-termo cada vez com menor peso (PEIXOTO, et al., 2002; RUGOLO, 2003). A vulnerabilidade biológica associada a imaturidade neurológica e fisiológica coloca estes bebés em risco de desenvolverem complicações ao longo do desenvolvimento, tais como paralisia cerebral, deficiências auditivas e visuais e perturbações de linguagem. Neste contexto, torna-se relevante reflectir sobre o futuro destes bebés, a curto e a longo prazo, e perceber de que forma o desenvolvimento global e linguístico destas crianças ocorre, para que se possam tomar, atempadamente, as medidas necessárias no sentido da preservação de um crescimento harmonioso.

Vários investigadores têm demonstrado interesse pela pesquisa sobre o impacto das variáveis perinatais peso e idade gestacional ao longo do desenvolvimento infantil (ALS, 1997; HUANG, et al., 2012; VOIGT, et al., 2012; VOHR; MSALL, 2004). Diversos estudos relatam as características das crianças nascidas com muito baixo peso e as dificuldades por elas experienciadas. Contudo, o foco de atenção encontra-se na pesquisa relativa ao desenvolvimento infantil global e à identificação de patologias, existindo, ainda, fortes lacunas na investigação em áreas mais específicas como a do desenvolvimento linguístico.

Vários estudos internacionais referem o baixo peso como factor preditivo do desenvolvimento de défices no domínio da linguagem (ALLEN, 2002; HANKE, et al., 2003; LUOMA, et al., 1998; MIKKOLA, et al., 2005; SAJANIEMI, et al., 2001). No entanto, embora em Portugal a prevalência de partos pré-termo tenha vindo a 
aumentar no decorrer dos anos, não existem estudos sobre a relação entre estes e a presença de défices linguísticos. Tal facto torna pertinente a implementação de pesquisas que permitam caracterizar o percurso de desenvolvimento dos recémnascidos com muito baixo peso em Portugal e, consequentemente, perceber se a variável muito baixo peso pode ser considerada um factor de risco biológico para o desenvolvimento de défices linguísticos.

Em síntese, e na sequência dos aspectos supramencionados, o objectivo central deste estudo é o de identificar possíveis diferenças de desenvolvimento fonológico entre os dois grupos de crianças sob avaliação (crianças nascidas com muito baixo peso e crianças nascidas com peso adequado), testando a eficácia das variáveis fonológicas apontadas na literatura como as mais relevantes para a caracterização do perfil fonológico infantil. Assim, e tendo em consideração os vários aspectos referidos nesta secção, o presente estudo pretende caracterizar as competências fonológicas de crianças entre os 3 anos e 6 meses e os 4 anos e 6 meses $^{11}$ nascidas com muito baixo peso e verificar se os valores obtidos por este grupo diferem dos atingidos por crianças com peso adequado. Considerar-se-á, para o efeito, o inventário segmental do PE (todas as entidades fonológicas que integram as classes naturais das consoantes oclusivas, das nasais, das fricativas e das líquidas); estes segmentos serão testados em função das variáveis posição na

palavra, posição na sílaba e acento de palavra. Desta forma, observar-se-á o grau de produtividade destas estruturas na avaliação clínica, discutindo-se a sua eficácia como indicadores do perfil fonológico de crianças nascidas com muito baixo peso, quando comparadas com crianças nascidas com peso adequado.

\section{METODOLOGIA}

\section{PARTICIPANTES}

Participaram, neste estudo, 30 crianças de ambos os géneros, com idades compreendidas entre os 3;6 e os 4;6, todas residentes na zona noroeste do país.

A população de recém-nascidos com muito baixo peso (RNMBP) foi composta por quinze indivíduos; os dados foram recolhidos num hospital público da área do Porto. As crianças deste grupo preenchiam os seguintes critérios de inclusão: (i) nascidas no hospital seleccionado para o estudo e inscritas na consulta de desenvolvimento; (ii) peso à nascença inferior a 1500g; (iii) idade, à data da recolha, entre os 3;6 e os 4;6; (iv) monolíngues do PE; (v) sem intervenção em terapia da fala e sem história de patologias neurosensoriais e motoras. Neste grupo experimental, foi considerado como factor de exclusão o encaminhamento dos recém-nascidos para uma zona de residência fora do concelho do hospital em estudo logo após

A partir de agora, as idades passarão a ser representadas no formato 3;6 (3 anos e 6 meses). 
o nascimento, dada a ausência de registo do seu desenvolvimento clínico após o nascimento, o que impossibilitaria determinar quais os casos que responderiam aos critérios de inclusão e de exclusão do estudo.

Para as crianças do grupo de controlo, nascidas com peso adequado (RNPA), foram usados os seguintes critérios de inclusão: (i) peso adequado à nascença (>2500g); (ii) idade, à data da recolha, entre os 3;6 e os 4;6; (iii) monolingues do PE; (iv) ausência de história de patologias sensoriais e motoras e ausência de intervenção em terapia da fala.

A distribuição em função da variável género foi idêntica, sendo 53\% das crianças do género feminino e as restantes $47 \%$ do género masculino. A idade média e a variabilidade de idades (D.p) das crianças nascidas a termo no momento da avaliação e a das crianças nascidas com muito baixo peso foram, como seria de esperar, idênticas.

Relativamente ao peso à nascença, variável em análise no nosso estudo, toda a amostra do grupo de controlo possuía um peso superior a 2500g. No que diz respeito às crianças do grupo experimental, 60\% apresentava um peso de 1001 a 1501g e 40\% apresentava um peso inferior a 1000g. O peso médio dos RNMBP era claramente inferior ao dos RNPA. Os restantes dados de caraterização da amostra são apresentados na tabela que se segue (Tabela 1):

Tabela 1 - Dados de caraterização da amostra

\begin{tabular}{|c|c|c|c|}
\hline & & $\begin{array}{l}\text { Grupo de muito } \\
\text { baixo peso }\end{array}$ & $\begin{array}{l}\text { Grupo peso } \\
\text { adequado }\end{array}$ \\
\hline Comprimento & Média $\pm \mathrm{DP}$ & $35,62 \pm 4,90$ & $49,42 \pm 1,09$ \\
\hline Perímetro Cefálico & Média \pm DP & $26,12 \pm 2,82$ & $34,67 \pm 1,28$ \\
\hline Peso & Média \pm DP & $1063,33 \pm 278,42$ & $3420 \pm 318,42$ \\
\hline Idade gestacional & Média $\pm \mathrm{DP}$ & $29,13 \pm 2,79$ & $39,2 \pm 1,08$ \\
\hline $\begin{array}{l}\text { Idade (meses) momento } \\
\text { da avaliação }\end{array}$ & Média \pm DP & $48,4 \pm 3,69$ & $49,53 \pm 3,96$ \\
\hline
\end{tabular}

Fonte: Tabela original decorrente do estudo. 
Para todas as crianças, foi preenchida uma ficha de caracterização da amostra, com vista à recolha uniforme dos dados clínicos e sócio-demográficos (NOGUEIRA, 2007).

\section{INSTRUMENTO}

Foi construído um instrumento de avaliação do desenvolvimento fonológico infantil, criado especificamente para o presente estudo, com o formato de teste de nomeação e com palavras-alvo da categoria nome. A construção do instrumento de trabalho teve por base as relações entre os segmentos consonânticos e as variáveis posição na palavra, posição na sílaba e acento de palavra, estudadas no domínio da investigação sobre aquisição da fonologia típica e atípica, como vimos na secção inicial deste artigo (o instrumento é integralmente apresentado em NOGUEIRA, 2007). Consideraram-se todas as consoantes fonológicas do PE padrão (oclusivas [p, b, t, d, k, g], nasais [m, n, n], fricativas [f, v, S, z, x, j] e líquidas $[l, K, r, R])$; a sua ocorrência nos estímulos lexicais apresentados foi controlada em função de (ilustração com a lateral /l/):

(i) os constituintes silábicos a que podem estar associadas (Ataque simples (lápis); Ataque ramificado (flor) e Coda (soll));

(ii) a posição que podem ocupar na palavra (Ataque: posição inicial (lápis) ou posição medial (gelado); Coda: posição medial (calças) ou posiç̧ão final (soln));

(iii) o contexto acentual em que podem ocorrer (tónico (lápis); átono (limão)).

Procurou-se que os estímulos lexicais seleccionados:

(i) fossem de representação visual inequívoca;

(ii) fizessem parte do léxico infantil na faixa etária testada (FREITAS, 1997);

(iii) fossem identificados por um número alargado de crianças, previamente testadas para o efeito;

(iv) sempre que possível, tivessem sido utilizados por outros investigadores, em testes de avaliação linguística (GUIMARÃES; GRILO, 1996; FARIA; FALÉ, 2001).

Previamente à definição do formato final do instrumento de avaliação fonológica usado na presente investigação, foi efectuado um pré-teste com formato de teste de nomeação, com vista à selecção dos estímulos não problemáticos dos pontos de vista visual e lexical. Nesta etapa, utilizou-se um número de estímulos (71 imagens) superior ao final (56 imagens), com os seguintes objectivos:

(i) identificar os mais facilmente nomeados pelas crianças, de entre os considerados como representativos das estruturas fonológicas em foco (estímulos lexicais distintos que avaliavam uma mesma consoante num mesmo contexto fonológico); 
(ii) nos casos de estímulos não usados noutros testes de avaliação linguística, testar mais do que uma imagem para representar um mesmo alvo lexical, com o objectivo de avaliar a eficácia da imagem na tarefa solicitada.

O teste de nomeação foi aplicado a oito crianças com idades semelhantes às da amostra do estudo, que se encontravam integradas num jardim-de-infância da zona do Porto. Através da identificação das percentagens de acerto relativamente a cada imagem integrada no teste de nomeação, tornou-se possível proceder à selecção das imagens presentes no instrumento final de avaliação fonológica usado nesta investigação. Para a integração de cada estímulo no instrumento de avaliação fonológica construído, foi usado o critério de inclusão de, no mínimo, 75\% de acerto na nomeação de cada estímulo visual apresentado, para garantir o sucesso no reconhecimento das imagens no estudo principal. No caso de 3 palavras (grilo, formiga, túnel), foi violado este critério quantitativo: a taxa de sucesso na sua identificação foi inferior a $75 \%$ e superior a 50\%, mas, dada a impossibilidade de as substituir por outros itens lexicais com a mesma estrutura fonológica e possíveis no léxico infantil, estes três estímulos foram mantidos na versão final do instrumento.

Sempre que possível, um mesmo estímulo foi usado para avaliar mais do que uma estrutura fonológica, de forma a integrar o menor número de estímulos possível no instrumento de avaliação construído: a título ilustrativo, a palavra "castelo" avalia o /t/ tónico medial em Ataque simples e o / / / em Coda átona medial.

\section{PROCEDIMENTOS}

A recolha de dados para o presente estudo teve início com o pedido de autorizações à Administração e à Comissão de Ética do hospital, bem como às instituições de ensino das crianças observadas, explicitando-se os objectivos do estudo e os procedimentos a levar a cabo. Os pais foram esclarecidos quanto à metodologia de trabalho e sua utilidade, sendo novamente assegurado o carácter voluntário da sua participação. Apenas as crianças cujos pais concordaram com as condições de realização do estudo, através da assinatura do consentimento informado, foram incluídas na amostra final.

A recolha de dados junto do grupo experimental foi efectuada nas instalações do hospital usado para a recolha de dados, em dois momentos. Realizou-se um levantamento de dados que abrangeu todas as crianças nascidas na Unidade de Cuidados Intensivos Neonatais (UCIN) do hospital com peso inferior a $1500 \mathrm{~g} \mathrm{e}$ na faixa etária dos 3;6 aos 4;6, à data da recolha de dados.

A constituição do grupo de controlo foi obtida, por conveniência, num Jardim de Infância do distrito de Viana do Castelo, onde foram seleccionadas crianças 
passíveis de serem emparelhadas com as do grupo experimental, através das variáveis idade e género. A recolha de dados decorreu igualmente em dois momentos distintos do mesmo ano.

As crianças do grupo experimental foram avaliadas em gabinete hospitalar; as do grupo de controlo, numa sala privada no Jardim de Infância. A cada criança era

solicitado que nomeasse espontaneamente cada uma das imagens apresentadas isoladamente; no caso de não haver nomeação espontânea, eram fornecidas à criança pistas semânticas com vista à estimulação da nomeação. As produções foram registadas, através de transcrição fonética, em folha de registo construída para o efeito e, simultaneamente, gravadas em formato áudio, através de um gravador Philips AD751-Speech Trainer Recorder, com uso de um microfone externo unidireccional.

\section{TRATAMENTO DOS DADOS}

O material linguístico recolhido foi transcrito foneticamente, primeiro, em folhas de registo, no momento da recolha de dados, e, posteriormente, a partir das gravações áudio efectuadas. Para cada estímulo, foram confrontadas as duas transcrições de cada estímulo e, em caso de não convergência de ambas as transcrições, optou-se por solicitar o apoio de um outro Terapeuta da Fala, que funcionou como juiz na avaliação das transcrições.

Os dados recolhidos foram organizados numa base de dados em formato Software Access, da Microsoft. Em seguida, os dados contidos na base e a informação da ficha de caracterização foram seriados e editados no programa SPSS forWindows - versão 13, para tratamento estatístico. Tendo em conta os objectivos do estudo e a natureza dos dados recolhidos, usaram-se os procedimentos da estatística descritiva para a obtenção da média e do desvio padrão relativos aos parâmetros sócio-demográficos e às frequências de produção das consoantes em função das diferentes variáveis fonológicas estudadas (posição na sílaba, posição na palavra e acento de palavra). Ao longo do trabalho, a frequência de produção foi relacionada com o grau de estabilidade dos segmentos e/ou das classes naturais estudados (BERNHARDT; STEMBERGER, 1998; FIKKERT, 1994; FREITAS, 1997; INGRAM, 1999).

Quando foi necessário proceder a uma análise composta por mais do que um segmento, formaram-se agregados através do somatório de produções dos diferentes segmentos em estudo. A partir deste somatório, formaram-se intervalos consoante o número de segmentos agregados. No conjunto de agregados, elaboraram-se tabelas de frequências cruzadas ou tabelas de contingência, que permitiram estudar a relação entre duas variáveis categóricas, descrevendo a frequência das categorias de uma das variáveis relativamente à das categorias de outra. 


\section{RESULTADOS}

Nesta seção, serão apresentados os resultados obtidos para grupo experimental e para grupo de controlo, através da aplicação do instrumento de avaliação fonológica construído.

Considere-se a taxa global de sucesso por classe do modo de articulação (consoantes oclusivas, nasais, fricativas e líquidas) na produção de consoantes em Ataque simples, constituinte silábico no qual ocorrem todas as consoantes do PE. Como podemos observar na Figura 1, as oclusivas orais e nasais são as primeiras a estabilizar no processo de desenvolvimento linguístico das crianças observadas, embora as nasais apresentem um nível de sucesso inferior ao das orais. Os valores de produção com sucesso descem ainda mais na classe das fricativas, acentuando-se esta descida na classe das líquidas (vibrantes e laterais). Tendo em conta a comparação entre grupos, torna-se possível verificar que os dois grupos seguem o mesmo percurso de aquisição, embora o grupo dos RNMBP apresente valores de produção inferiores aos do grupo dos RNPA nas classes mais problemáticas (fricativas, vibrantes e laterais).

Figura 1 - Classes naturais em Ataque simples por grupo (RNMBP e RNPA)

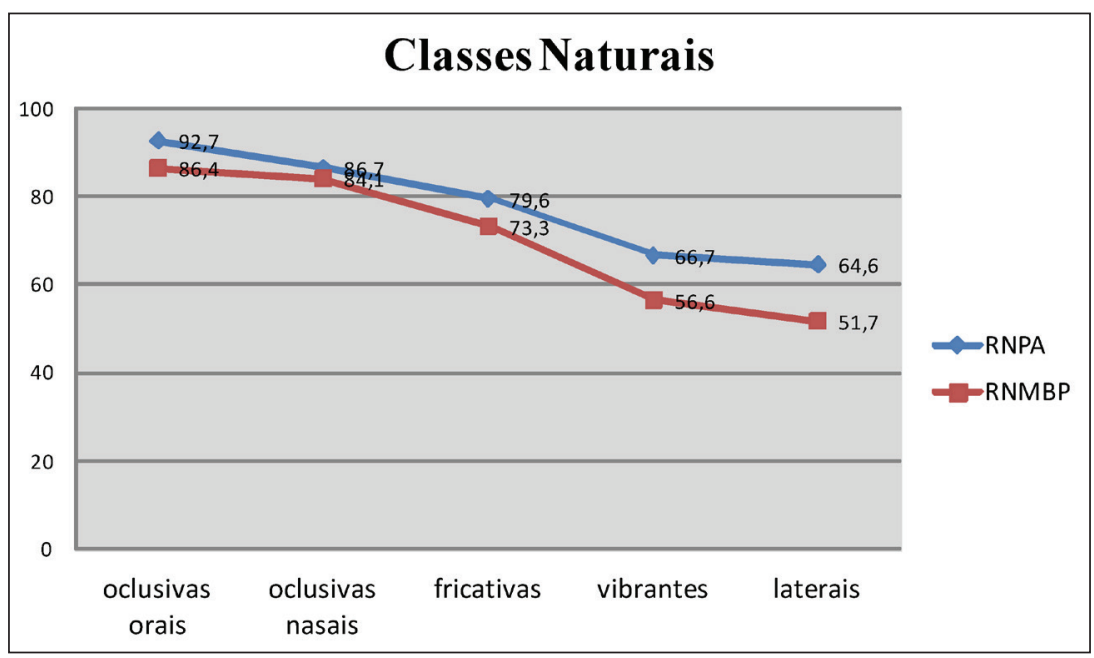

Fonte: Figura original decorrente do estudo.

As Tabelas 2 e 3, abaixo, apresentam resultados para as classes naturais do modo de articulação estudadas, quando consideradas apenas as consoantes em Ataque simples em função das variáveis posição na palavra e acento de palavra. 
Relativamente à variável posição na palavra, as crianças do grupo RNPA apresentaram valores de desempenho tendencialmente superiores aos do grupo de crianças RNMBP: vejam-se os valores para as oclusivas orais, para as fricativas e para as líquidas na Tabela 2, sendo a classe das oclusivas nasais a única que não permite discriminar comportamentos entre os dois grupos. Ainda no que diz respeito ao papel desta variável no desempenho linguístico das crianças observadas: (i) no grupo de controlo (RNPA), a posição inicial demonstrou ser um contexto tendencialmente facilitador do sucesso na produção segmental (cf. resultados para as oclusivas orais, para as nasais e para as líquidas); (ii) já no grupo experimental (RNMBP), esta variável não desempenhou um papel promotor do sucesso no desempenho segmental.

Tabela 2 - Variável posição na palavra por grupo (RNMBP e RNPA)

\begin{tabular}{c|c|c|c}
\hline $\begin{array}{c}\text { Posição na } \\
\text { palavra }\end{array}$ & RNMBP & RNPA \\
\hline \multirow{2}{*}{ Oclusivas orais } & Inicial & $60 \%$ & $80 \%$ \\
\cline { 2 - 4 } & Medial & $60 \%$ & $73 \%$ \\
\hline \multirow{2}{*}{ Oclusivas nasais } & Inicial & $87 \%$ & $80 \%$ \\
\cline { 2 - 4 } & Medial & $60 \%$ & $60 \%$ \\
\hline \multirow{2}{*}{ Fricativas } & Inicial & $40 \%$ & $67 \%$ \\
\cline { 2 - 4 } & Medial & $53 \%$ & $67 \%$ \\
\hline \multirow{2}{*}{ Líquidas } & Inicial & $40 \%$ & $80 \%$ \\
\cline { 2 - 4 } & Medial & $40 \%$ & $53 \%$ \\
\hline
\end{tabular}

Fonte: Tabela original decorrente do estudo.

No que diz respeito à variável acento de palavra (confira Tabela 3), e tendo em conta a comparação entre grupos, uma vez mais o grupo de RNPA apresentou valores superiores aos do grupo de RNMBP (excepto na classe das oclusivas nasais). Quanto ao papel promotor da posição tónica no desempenho verbal infantil, há a registar diferenças em função do grupo em análise: (i) no grupo dos RNPA, as sílabas tónicas parecem ser contextos facilitadores para a produção das oclusivas, das fricativas e das líquidas, não havendo diferença a registar nas nasais; no grupo experimental (RNMBP), o efeito promotor da variável acento de palavra não é claro, pois as sílabas tónicas promovem as fricativas mas não as oclusivas orais (valores iguais nos dois contextos), as nasais e as líquidas (nestes dois casos, valores superiores nas átonas). 
Tabela 3 - Variável acento de palavra por grupo (RNMBP e RNPA)

\begin{tabular}{cccc}
\hline Contexto acentual & & RNMBP & RNPA \\
\hline \multirow{2}{*}{ Oclusivas orais } & Tónico & $60 \%$ & $80 \%$ \\
\cline { 2 - 4 } & Átono & $60 \%$ & $67 \%$ \\
\hline \multirow{2}{*}{ Oclusivas nasais } & Tónico & $87 \%$ & $80 \%$ \\
\cline { 2 - 4 } & Átono & $93 \%$ & $80 \%$ \\
\hline \multirow{2}{*}{ Fricativas } & Tónico & $67 \%$ & $80 \%$ \\
\cline { 2 - 4 } & Átono & $53 \%$ & $53 \%$ \\
\hline \multirow{2}{*}{ Líquidas } & Tónico & $40 \%$ & $80 \%$ \\
\cline { 2 - 4 } & Átono & $67 \%$ & $73 \%$ \\
\hline
\end{tabular}

Fonte: Tabela original decorrente do estudo.

Em Coda, o inventário segmental em PE é muito restrito, sendo apenas possíveis quatro segmentos fonéticos. O instrumento construído para a recolha de dados permite testar estas estruturas (Coda fricativa, Coda lateral, Coda vibrante) nas posições tónica e átona, medial e final. Podemos observar, nas Figuras 2 e 3 , o desempenho dos dois grupos de crianças nos diferentes contextos testados.

A Figura 2 apresenta resultados relativos à produção da Coda fricativa, da Coda lateral e da Coda vibrante nos diferentes contextos acentuais (tónico, átono).

Figura 2 - Desempenho dos grupos nas

Codas em função da variável acento de palavra

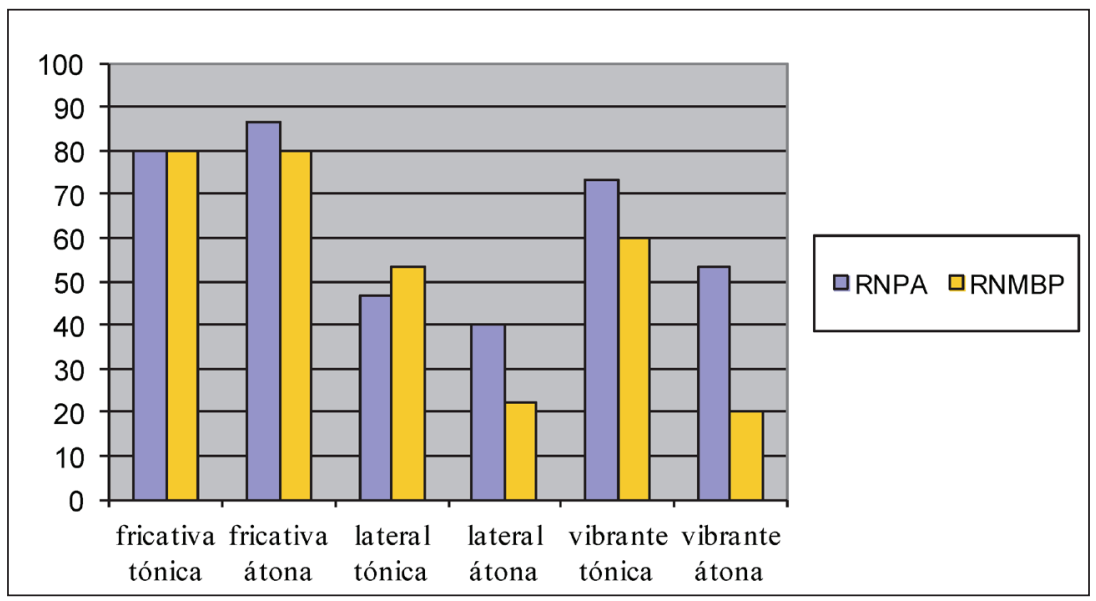

Fonte: Figura original decorrente do estudo. 
De uma forma geral, podemos constatar que a Coda fricativa apresenta, em ambos os grupos, valores percentuais de produção superiores aos registados para as Codas líquidas, o que demonstra um comportamento mais estável face à primeira. Quanto à comparação entre grupos, os valores de sucesso na produção da Coda fricativa são iguais nos dois grupos de sujeitos sob avaliação; em posição átona, os valores do grupo experimental (RNMBP) são ligeiramente inferiores aos do grupo de controlo.

Já na classe das líquidas, o comportamento é mais heterogéneo. Em ambos os casos (Coda lateral e Coda vibrante), a posição tónica parece promover o sucesso na produção, registando-se valores mais elevados para a vibrante do que para a lateral. No que diz respeito ao confronto entre grupos, excepto no caso da Coda lateral tónica, os restantes contextos (Coda lateral átona, Coda vibrante tónica e Coda lateral átona) são mais problemáticos para os RNMBP do que para os RNPA.

De acordo com os resultados na Figura 2, as consoantes em Coda revelam-se, assim, bons preditores do desenvolvimento fonológico, quer dentro de cada grupo, quer no confronto entre grupos, sendo registada a seguinte ordem de aquisição: Coda fricativa $>>$ Coda vibrante $>>$ Coda lateral.

Como referido no início deste artigo, o sucesso na produção das Codas parece depender da sua posição na palavra. No caso do PE, a posição final de palavra tem-se revelado promotora da aquisição desta estrutura. Na Figura 3, é possível comparar o desempenho dos dois grupos de sujeitos sob avaliação relativamente aos três tipos de Codas nas duas posições da palavra (medial e final).

Figura 3 - Desempenho dos grupos nas Codas em posição final e medial

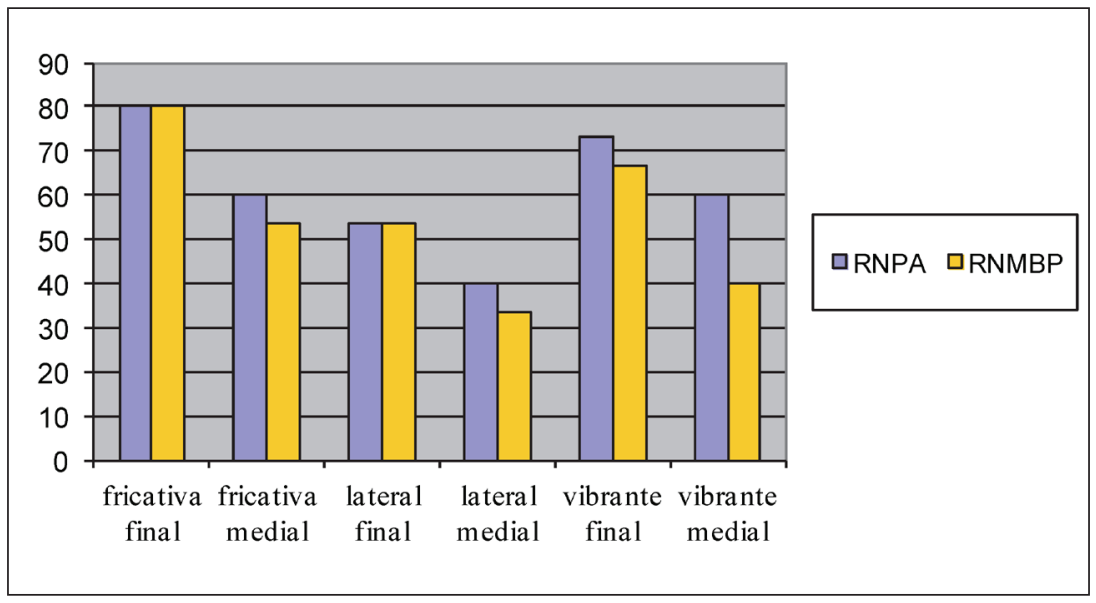

Fonte: Figura original decorrente do estudo. 
Para cada um dos três tipos de Coda (fricativa, lateral e vibrante), a posição final favorece a produção da estrutura-alvo: em cada tipo de Coda, os valores são sempre mais altos em posição final do que em posição medial. Esta variável revela-se, assim, produtiva na predição do desenvolvimento fonológico infantil.

Ao confrontarmos os dados dos dois grupos de crianças na Figura 3, verificamos que os RNMBP tendem a apresentar níveis de sucesso na produção inferiores aos dos RNPA. Em termos globais, e tendo em conta os dados registados nas Figuras 2 e 3, com excepção da Coda lateral tónica final e da Coda fricativa tónica final, todas as outras variáveis apresentam valores percentuais inferiores no grupo de MBP, do que no grupo de peso adequado.

Com vista à observação do impacto da variável constituinte silábico nas produções dos grupos testados, centremo-nos agora na produção das fricativas palatais e das líquidas alveolares, por serem os segmentos do PE que podem ocorrer nas três posições silábicas que hospedam consoantes: Ataque simples, Coda e Ataque ramificado. Através dos dados médios obtidos ao longo da presente pesquisa, expostos na Tabela 4, podemos verificar que a fricativa apresenta valores de sucesso idênticos nas duas posições silábicas (Ataque simples e Coda) e em ambos os grupos testados. Quanto à vibrante, existem diferenças de estabilização do segmento em função do grupo avaliado (os RNMBP apresentam valores mais baixos do que os RNPA) e em função dos diferentes constituintes silábicos (os níveis de sucesso na produção estão organizados de acordo com a seguinte escala decrescente: Ataque simples $>>$ Coda $>>$ Ataque ramificado). No caso da lateral, os valores para os RNMBP também são mais baixos do que os registados para os RNPA, mantendo-se a escala decrescente de sucesso em função dos diferentes constituintes já registada para a vibrante (Ataque simples $>>$ Coda $>>$ Ataque ramificado).

Tabela 4 - Variável constituinte silábico por grupo (RNMBP e RNPA)

\begin{tabular}{lcccccc}
\hline & \multicolumn{2}{c}{ Ataque simples } & \multicolumn{2}{c}{ Coda } & \multicolumn{2}{c}{ Ataque ramificado } \\
\hline & RNPA & RNMBP & RNPA & RNMBP & RNPA & RNMBP \\
\hline Fricativa & $76,67 \%$ & $76,67 \%$ & $78,89 \%$ & $75,56 \%$ & & \\
\hline Vibrante & $66,67 \%$ & $60,00 \%$ & $62,22 \%$ & $42,22 \%$ & $33,33 \%$ & $20,00 \%$ \\
\hline Lateral & $80,00 \%$ & $66,67 \%$ & $31,67 \%$ & $28,33 \%$ & $20,00 \%$ & $13,33 \%$ \\
\hline
\end{tabular}

Fonte: Tabela original decorrente do estudo. 
Em suma, os resultados recolhidos no âmbito do presente estudo e expostos nesta secção demonstram que os RNMBP em estudo tendem a apresentar um desempenho verbal global inferior ao do grupo de RNPA, no que se refere aos aspectos fonológicos tratados:

(i) no caso das classes naturais do modo de articulação, e considerando apenas o constituinte silábico Ataque simples, ambos os grupos de crianças revelaram a mesma ordem de aquisição; no caso das classes mais problemáticas (fricativas, vibrantes e laterais), o desempenho do grupo experimental (RNMBP) foi inferior ao do grupo de controlo (RNPA);

(ii) no caso da variável posição na palavra, e quando considerados os Ataque simples iniciais e mediais, os RNPA apresentaram valores de desempenho tendencialmente superiores aos dos RNMBP, sendo a posição inicial um contexto promotor nos RNPA mas não nos RNMBP; no caso das Codas, a posição final revelou-se mais facilitadora da produção do que a medial, em ambos os grupos de sujeitos, registando-se, globalmente, valores de sucesso mais baixos nos RNMBP do que nos RNPA;

(iii) quanto à variável acento de palavra, e considerando apenas as consoantes em Ataque simples, os RNPA apresentaram valores tendencialmente superiores aos dos RNMBP; esta variável revelou-se promotora do desenvolvimento nos RNPA mas não nos RNMBP; no caso das Codas problemáticas (lateral e vibrante), o acento de palavra constituiu uma variável produtiva na avaliação do desempenho, com a posição tónica a favorecer o comportamento em ambos os grupos, revelando os RNPA mais sucesso do que os RNMBP;

(iv) no que diz respeito à variável posição na sílaba, e tendo em conta os segmentos $/ 1 /$ e $/ \mathrm{f} /$, foi identificada a ordem de aquisição Ataque simples $>>$ Coda $>>$ Ataque ramificado, sendo os resultados para as fricativas idênticos nos constituintes Ataque e Coda; os valores de sucesso na produção de cada um dos segmentosalvo são sempre mais baixos nos RNMBP do que nos RNPA.

\section{CONSIDERAÇÕES FINAIS}

Neste artigo, retomámos algumas das variáveis fonológicas mais trabalhadas no domínio da aquisição fonológica típica e atípica - posição na palavra, posição na sílaba e acento de palavra (FIKKERT, 1994; FREITAS, 1997; BERNHARDT; STEMBERGER, 1998; LAMPRECHT, 1999; ROSE, 2000; SANTOS, 2001; LAMPRECHT, et al., 2004; KAGER; PATER; ZONNEVELD, 2004; DEMUTH, 2009; ALMEIDA, 2011; BERNHARDT; STEMBERGER, 2000), com o objectivo de testar a sua produtividade na avaliação de um grupo de crianças nascidas com muito baixo peso (grupo experimental - RNMBP), quando comparadas com crianças nascidas com peso adequado (grupo de controlo - RNPA). 
Em termos globais, a presença de valores de sucesso mais baixos no grupo experimental, nas várias estruturas fonológicas testadas, revelaram um desempenho verbal mais imaturo nos RNMBP, quando comparados com os RNPA. Diversos estudos têm relatado que crianças nascidas com muito baixo peso apresentam maior predisposição para desenvolver défices do desenvolvimento linguístico. Autores como Montgomery et al. (1997), Largo et al. (1986) e Luoma et al. (1998) referem que os RNMBP manifestam, ao longo das avaliações, resultados inferiores aos seus pares nascidos com peso adequado. Segundo os mesmos autores, estas crianças apresentam um desempenho mais fraco nos testes aplicados, evidenciando um atraso no desenvolvimento linguístico. Os dados apresentados no presente estudo vão, assim, ao encontro dos descritos nestes estudos. Note-se, no entanto, que, nos vários contextos fonológicos estudados, os dois grupos de crianças testados apresentaram a mesma ordem de aquisição das estruturas em foco, o que aponta, não para a identificação de perturbações, mas sim para um atraso no desenvolvimento fonológico dos RNMBP relativamente aos RNPA (MONTGOMERY, et al., 1997; SAJANIEMI, et al., 2001). Este atraso pode ser, em parte, explicado por uma mielinização incompleta e, consequentemente, uma interconexão entre os neurónios das áreas secundárias mais tardia, que caracteriza os RNMBP. Estes processos neurológicos, responsáveis, entre outros aspectos, pela reorganização das categorias acústicas e processamento dos esquemas fonoarticulatórios específicos de uma determinada língua, podem afectar o domínio das representações mentais dos gestos articulatórios, levando a um atraso no desenvolvimento fonológico (PEIJEN, et al., 2008; ZOMIGNANI, et al., 2009).

As variáveis linguísticas estudadas revelaram-se bons indicadores do desenvolvimento fonológico no grupo de crianças nascidas a termo, sendo mais evidente o efeito da variável posição na sílaba, quando comparada com as variáveis posição na palavra e acento de palavra. Relativamente às diferenças entre os dois grupos avaliados, embora os RNMBP adquiram os segmentos mais tardiamente, atraso que tende a acentuar-se em tarefas e estádios mais complexos (cf. aquisição de /1/ e /r/ em diferentes posições silábicas), o teste do Qui-quadrado não identificou diferenças estatisticamente significativas entre os grupos.

Tendo em consideração apenas os dados relativos às consoantes em Ataque simples, constituinte silábico que pode hospedar todas as consoantes do $\mathrm{PE}$, ambos os grupos observados neste trabalho revelaram a seguinte ordem de aquisição das classes naturais em foco: oclusivas orais $>>$ oclusivas nasais $>>$ fricativas $>>$ líquidas. Tal ordem de aquisição vai ao encontro do relatado para várias línguas, com as oclusivas orais e as nasais a serem adquiridas precocemente, após o que se adquirem as fricativas e só mais tarde as líquidas (FIKKERT, 1994; BERNHARDT; STEMBERGER, 1998; JOHNSON; REIMERS, 2010). 
No entanto, no caso do PE e do Português do Brasil (PB), tem sido relatado o facto de as oclusivas orais e as nasais serem adquiridas simultaneamente (FREITAS, 1997; LAMPRECHT, et al., 2004; COSTA, 2010), o que não faria prever diferenças entre estas duas classes naturais nos dados observados. Validação empírica adicional poderá confirmar ou infirmar a diferença aqui detectada entre as duas classes de aquisição precoce. Note-se, no entanto, que a diferença de valores é reduzida e que, em ambas as classes, os níveis de sucesso estão acima dos 85\%, o que indicia a aquisição de ambas as classes no momento da avaliação. Na ordem de aquisição segmental das línguas naturais, existem dois percursos possíveis de aquisição para as classes mais problemáticas: um em que as fricativas seguem as oclusivas orais e nasais no processo de desenvolvimento; outro em que as líquidas antecedem as fricativas. O percurso encontrado na presente investigação vai ao encontro do referenciado na literatura como o mais comum no processo de aquisição, revelando as fricativas valores mais elevados do que as líquidas (FIKKERT, 1994).

A posição na palavra tem sido referida como uma variável relevante para o estudo da aquisição do inventário segmental de uma língua (LEVELT, 1994; FIKKERT, 1994; BERNHARDT; STEMBERGER, 1998; FREITAS, MIGUEL; FARIA, 2001; CORREIA, 2004; COSTA, 2010). No presente trabalho, e considerando apenas o constituinte Ataque simples (posições inicial $e$ medial), a posição inicial de palavra revelou-se maioritariamente promotora dos níveis de sucesso no grupo dos RNPA. Estes resultados vão ao encontro de diversos estudos que referem as estruturas no início de palavra como sendo adquiridas numa etapa mais precoce do desenvolvimento, tendendo os segmentos a emergir e a estabilizar mais tardiamente em posição medial (LEVELT, 1994; COSTA, et al., 2007; FREITAS, et al., 2006). No entanto, no caso dos RNMBP, esta variável não se mostrou relevante para a avaliação da produção, com as várias classes naturais a serem alvo de comportamentos distintos. Note-se que nem todos os estudos corroboram a natureza promotora da posição inicial de palavra: Costa (2010) refere o facto de os pontos de articulação Labial e Dorsal em PE serem adquiridos primeiro em início de palavra, o que não acontece com a classe das líquidas, adquirida primeiro em posição medial. O impacto da variável posição na palavra (inicial ou medial) na avaliação do desenvolvimento segmental infantil em PE carece, assim, de investigação suplementar.

No caso da Coda, a observação dos resultados em função da variável posição na palavra mostraram o efeito promotor da posição final, quando comparada com a medial. Como referimos anteriormente, Freitas, Miguel e Faria (2001) e Correia (2004) relatam a aquisição precoce das Codas finais em PE, quando comparadas com as mediais. Estes resultados foram interpretados, no caso das Codas fricativas, como decorrentes do efeito promotor da interface gramatical fonologia-morfologia 
na periferia direita da palavra, uma vez que as Codas fricativas em final de palavra transportam, regularmente, informação de natureza flexional: (i) marcam o número plural no sistema nominal (pato/patos); (ii) marcam pessoa/número no sistema verbal (falo, falas, fala). Nos dados relatados no presente estudo, a posição final revelou-se mais promotora da produção do que a medial, o que vai ao encontro do relatado na literatura sobre o $\mathrm{PE}$, sendo os valores tendencialmente mais baixos nos RNMBP do que nos RNPA.

Os resultados relativos à variável acento de palavra mostraram que, nos RNPA, os Ataques simples tónicos apresentam taxas de sucesso mais altas do que os átonos. Através do cruzamento de dados relativos a esta variável, observou-se que as crianças deste grupo produzem alguns segmentos em posição tónica que ainda não são capazes de produzir em posição átona. Estes dados vão ao encontro do relatado em diversos estudos, corroborando o efeito promotor do acento de palavra na aquisição do inventário segmental (FIKKERT, 1994; FREITAS, 1997; MATZENAUER, 2001; KENT, 2004; SMITH, 2004; CORREIA, 2004). Em contrapartida, o grupo experimental exibiu um comportamento instável relativamente à variável em foco, não revelando o efeito atestado no grupo de controlo (registe-se apenas a excepção relativa às fricativas). A variável acento de palavra revelou-se, assim, produtiva na discriminação dos dois grupos avaliados, com inesperada anulação, no grupo experimental, do efeito do acento na aquisição das classes de consoantes testadas. A precoce sensibilidade prosódica das crianças, fortemente documentada na literatura (MORGAN; DEMUTH, 1996; JUSCZYK, 1997; GUASTI, 2002; FIKKERT, 2007; DEMUTH, 2009), e a aquisição também precoce do acento de palavra não fariam prever os resultados obtidos para os RNMBP. Em que medida estes resultados poderão ou não estar relacionados com a imaturidade do sistema linguístico das crianças nascidas com muito baixo peso constitui tópico de investigação futura. No entanto, no caso específico das Codas mais problemáticas (a Coda lateral e a Coda vibrante), o acento parece constituir uma variável produtiva na avaliação do desempenho fonológico tanto dos RNPA como dos RNMBP, com a posição tónica a favorecer o sucesso na produção, quando comparada com a posição átona.

O impacto dos constituintes silábicos na ordem de aquisição das consoantes de uma dada língua encontra-se amplamente documentado na literatura (FIKKERT, 1994; FREITAS, 1997; ROSE, 2000; KIRK; DEMUTH, 2003; LAMPRECHT, et al., 2004; ALMEIDA, 2011). No presente trabalho, procedeu-se à avaliação da variável posição na sílaba através da observação dos segmentos que exibem diferentes estatutos silábicos no PE: as fricativas palatais (Ataque simples e Coda), a vibrante alveolar e a lateral alveolar (Ataque simples, Coda e Ataque ramificado). As fricativas mostraram taxas de sucesso idênticas em ambos os constituintes, o que se justifica pela idade das crianças testadas: aos 3 anos e 6 
meses, idade de início da avaliação, esta classe já apresenta um comportamento estável em ambas as posições silábicas (FREITAS, 1997; CORREIA, 2004).

Nos casos de /l/ e /r/, foi identificada a ordem de aquisição Ataque simples $>>$ Coda $>>$ Ataque ramificado, consistente com os resultados disponíveis para o PE (FREITAS, 1997; ALMEIDA, 2011). O Ataque simples promove a aquisição das consoantes em foco, o que decorre da estrutura silábica universal CV (JAKOBSON, 1968), que exibe apenas Ataque simples. Contrariamente, o Ataque ramificado inibe a estabilização de /l/ e / / / até muito tarde, por ser, em várias línguas do mundo, o último constituinte disponibilizado no desenvolvimento fonológico infantil (FIKKERT, 1994; FREITAS, 1997; GNANADESIKAN, 2004; LAMPRECHT, et al., 2004).

No caso específico das Codas, os resultados revelaram a seguinte ordem de aquisição: Coda fricativa $>>$ Coda vibrante $>>$ Coda lateral. Esta cronologia de eventos vem ao encontro dos resultados já existentes para o PE: (i) em Freitas (1997), regista-se um forte contraste entre Codas fricativas e Codas líquidas (lateral e vibrante), o que legitima a discussão sobre a natureza silábica distinta de fricativas e de líquidas em final de sílaba (Coda versus Núcleo (FREITAS, 1997, para discussão da proposta)); (ii) o mesmo contraste foi relatado por Correia (2004), embora a autora tenha identificado diferenças individuais na aquisição das duas líquidas (algumas crianças desenvolveram mais rapidamente a Coda lateral; outras, a Coda vibrante). Evidência empírica adicional permitirá identificar a ordem preferencial de aquisição destas duas líquidas em Coda no PE, mostrando os resultados do presente estudo uma maior dificuldade associada a /1/.

Torna-se possível concluir, assim, que as variáveis linguísticas se revelaram bons indicadores do desenvolvimento fonológico no grupo de crianças nascidas a termo. No entanto, no grupo das crianças nascidas com muito baixo peso, esta função das variáves linguísticas em avaliação mostrou-se menos significativa. Não nos é possível colocar hipóteses sobre os eventuais factores subjacentes a esta diferença de resultados entre os dois grupos mas, na verdade, parece que os RNMBP revelam percursos diferentes e estratégias de reconstrução do input distintas das dos seus pares, que tendem a ser mais acentuadas em estádios de desenvolvimento mais avançados.

A implementação do presente estudo implicou a criação de um instrumento de avaliação do conhecimento fonológico, o que, desde logo, pode ser apontado como uma limitação do estudo. Acreditamos, no entanto, que a investigação relatada neste artigo contribui com evidência empírica para uma reflexão sobre a estrutura de futuros instrumentos de avaliação fonológica linguisticamente controlados, de forma a promover observações mais precisas em contextos de diagnóstico e de intervenção clínicos. 
A investigação na área da prematuridade e do baixo peso é ainda recente, o que suscita, desde logo, diversas dificuldades metodológicas na organização e planeamento de um desenho de investigação. A multiplicidade de factores linguísticos e extralinguísticos que podem interferir no desenvolvimento dos RNMBP, a dificuldade em identificar os aspectos individuais que interferem no desenvolvimento infantil das crianças em foco e a investigação lacunar no domínio da avaliação da linguagem em RNMBP fazem deste trabalho um estudo preliminar, a ser prosseguido em investigação futura.

NOGUEIRA, P. M.; FREITAS, M. J. Phonological development in children of 3 years and 6 months to 4 years and 6 months old born with very low weight. Alfa, São Paulo, v.58, n.3, p.677-702, 2014.

- ABSTRACT: The central aim of the current study is to evaluate the phonological development of children with very low birth weight, thus contributing with empirical evidence to the debate on the role of phonological variables in the assessment of child language development. A study based on production data from 30 children aged 3 and a half years to 4 and a half years was performed; 15 children were part of the population with very low birth weight and the remaining 15 were part of the population with appropriate birth weight; both groups were paired by gender and age. The children's segmental performance in the two groups studied was observed; the data analysis was based on the relationship between the production of each segmental consonant from the European Portuguese segmental inventory and its distribution as a function of the tested phonological variables position within the word, syllable constituency and word stress. The main purpose of the study was then to test the adequacy of these phonological variables to the identification of differences between the two groups of children's phonological development. By using the chi-square test and the frequency comparison, the main results showed that children with very low birth weight presented a lower verbal performance when compared to the normality pattern, with respect to the phonological variables under evaluation.

- KEYWORDS: Very low weight infants. Phonological development. Word. Syllable. Syllabic constituents. Word stress.

\section{REFERÊNCIAS}

ABAURRE, B.; GALVES, C.; SCARPA, E. A interface fonologia-sintaxe: evidências do PB para uma hipótese top-down na aquisição. In: SCARPA, E. (Org.). Estudos de prosódia. Campinas: Ed. da UNICAMP, 1999. p.285-323.

ALLEN, M. C. Preterm outcomes research: a critical component of neonatal intensive care. Mental Retardation and Developmental Disabilities Research Reviews, New York, n.8, p.221-233, 2002.

ALMEIDA, L. Acquisition de la structure syllabique en contexte de bilinguisme simultané Portugais-Français. 2011. 348f. Tese (Doutorado em Linguística) - Faculdade de Letras da Universidade de Lisboa, Lisboa, 2011. 
ALS, H. Earliest intervention for preterm infants in the newborn intensive care unit. In: GURALNICK, M. J. The effectiveness of early intervention. Baltimore: Paul H. Brookes Publishing, 1997. p.47-75.

ARCHIBALD, J. Phonological acquisition and phonological theory. Hillsdale: Lawrence Erlbaum Associates, 1995.

AVERY, P.; DRESHER, E. (Org.). Contrast in phonology: theory, perception, acquisition. Berlin: Mouton de Gruyter, 2008.

BALL, M. et al. (Org.). The handbook of clinical linguistics. Cambridge: Blackwell, 2008.

BAVIN, E. (Org.). The cambridge handbook on child language. Cambridge: Cambridge University Press, 2009.

BERNHARDT, B. H.; STEMBERGER, J. P. Workbook in nonlinear phonology for clinical applications. Austin: Pro-Ed, 2000.

. Handbook of phonology development: from the perspective of constraint-based non-linear phonology. San Diego: Academic Press, 1998.

BISHOP, D.; LEONARD, L. (Org.). Speech and language impairment in children: causes, characteristics, intervention and outcome. Hove: Psychology Press, 2000.

BISOL, L. (Org.). Introdução a estudos de fonologia do Português Brasileiro 4.ed. Porto Alegre: EDIPUCRS, 2005.

CLEMENTS, N. The geometry of phonological features. Phonology Yearbook, Cambridge, n.2, p.223-252, 1985.

CORREIA, S. A aquisição da rima em Português Europeu: ditongos e consoantes em final de sílaba. 2004. 210f. Dissertação (Mestrado em Linguística) Faculdade de Letras da Universidade de Lisboa, Lisboa, 2004.

COSTA, T. The acquisition of the consonantal system in European Portuguese: focus on place and manner features. 2010. 310f. Tese (Doutorado em Linguística) - Faculdade de Letras, Universidade de Lisboa, Lisboa, 2010.

COSTA, T. et al. Sobre o PA na periferia esquerda da palavra. In: LOBO, M.; COUTINHO, M. A. (Org.). Textos seleccionados do XXII Encontro Nacional da Associação Portuguesa de Linguística. Lisboa: Associação Portuguesa de Linguística, 2007. p.315-328.

DEMUTH, K. The prosody of syllables, words and morphemes. In: BAVIN, E. (Org.). Cambridge handbook on child language. Cambridge: Cambridge University Press, 2009. p.183-198. 
DINNSEN, D.; GIERUT, J. (Org.). Optimality theory, phonological acquisition and disorders. London: Equinox, 2008.

DUARTE, S. Relações de distância e de complexidade entre traços distintivos na generalização em terapia de desvios fonológicos. 2006. 326f. Dissertação (Mestrado em Linguística Aplicada) - Universidade Católica de Pelotas, Pelotas, 2006.

FARIA, I. H.; FALÉ, I. Fendas palatinas: estudo multidisciplinar. Lisboa: Colibri, 2001.

FAVA, E. (Org.). Clinical linguistics: theory and applications in speech pathology and therapy. Amsterdam: John Benjamins Publishing Company, 2002.

FIKKERT, P. Acquiring phonology. In:LACY, P. (Org.). Handbook of phonological theory. Cambridge: Cambridge University Press, 2007. p.537-554.

Getting sound structures in mind: acquisition bridging linguistics ans psychology. In: CUTLER, A. (Org.). Twenty-first century psycholinguistics: four cornerstones. Lawrence Erlbaum Associates, 2005. p. 43-56.

. On the acquisition of prosodic structure. Leiden: HIL, 1994.

FLETCHER, P.; MACWHINNEY, B. The handbook of child language. Cambridge: Blackwell, 1995.

FREITAS, M. J. Aquisição da estrutura silábica do português europeu. 1997. 396f. Tese (Doutorado em Linguística) - Faculdade de Letras da Universidade de Lisboa, Lisboa, 1997.

FREITAS, M. J. et al. Efeitos prosódicos e efeitos de frequência no desenvolvimento silábico em português europeu. In: ENCONTRO NACIONAL DA ASSOCIAÇÃO PORTUGUESA DE LINGUÍSTICA, 21., 2006, Lisboa. Actas... Lisboa:Associação Portuguesa de Linguística, 2006. p.39-412.

FREITAS, M. J.; MIGUEL, M.; FARIA, I. Interaction between prosody and morphosyntax: plurals within codas in the acquisition of European Portuguese. In: HOEHLE, B.; WEISSENBORN, J. (Org.). Approaches to bootstrapping: phonological, lexical, syntactic and neurological aspects of early language acquisition. Amsterdam: John Benjamins, 2001. v.2, p.45-58.

GNANADESIKAN,A. Markedness and faithfulness constraints in child phonology. In: KAGER, R.; PATER, J.; ZONNEVELD,W. (Org.). Constraints in phonological acquisition. Cambridge: CUP, 2004. p.73-108.

GOLDSMITH, J. (Ed.). Phonological acquisition: the handbook of phonological theory. Cambridge: Blackwell Pub, 1995. 
GUASTI, M. T. Language acquisition: the growth of grammar. Cambridge:The MIT Press, 2002.

GUIMARÃES, I.; GRILO, M. Teste de articulação verbal (TAV). Lisboa: Escola Superior de Saúde de Alcoitão, 1996.

HANKE, C. et al. Preschool development of very low birth weight children born 1994-1995. European Journal of Pediatrics, Berlin, v.162, n.3, p.159-164, 2003.

HUANG, J. et al. Inattention and development of toddler born in preterm and with low birth weight. Kaohsiung Journal of Medical Sciences, Taiwan, n.28, p.390-396, 2012.

INGRAM, D. Cross-linguistic phonological acquisition. In: BALL, M. et al. (Org.). The handbook of clinical linguistics. Cambridge: Blackwell, 2008. p.626-640.

First language acquisition: method, description and explanation. Melbourne: Cambridge University Press, 1999.

First language acquisition: method, description and explanation. Cambridge: CUP, 1989.

JAKOBSON, R. Child language, aphasia and phonological universals. The Hague: Mouton, 1968.

JOHNSON,W.; REIMERS, P. Patterns in child phonology. Edinburgh: EUP, 2010. JUSCZYK, P. The discovery of spoken language. Cambridge: MIT Press, 1997. KAGER, R.; PATER, J.; ZONNEVELD, W. (Org.). Constraints in phonological acquisition. Cambridge: Cambridge University Press, 2004.

KENT, R. Normal aspects of articulation. In: BERNTHAL, J. E.; BANKSON, N. W. Articulation and phonology disorders. 5.ed. Boston: Pearson, 2004. p.1-62.

KESKE-SOARES, M. Terapia fonoaudiológica fundamentada na hierarquia implicacional dos traços distintivos aplicada em crianças com desvios fonológicos. 2001. 223f. Dissertação (Doutoramento em Linguística) - Pontifícia Universidade Católica do Rio Grande do Sul, Porto Alegre, 2001.

KIRK, C.; DEMUTH, K. Onset/Coda asymmetries in the acquisition of clusters. In: BEACHLEY, B.; BROWN, A.; CONLIN, F. ANNUAL BOSTON UNIVERSITY CONFERENCE ON LANGUAGE DEVELOPMENT, 27., 2003, Sommerville. Proceedings... Sommerville: Cascadilla Press, 2003. p.437-448.

LAMPRECHT, R. R. Desvios fonológicos: evolução nas pesquisas, conhecimento atual e implicações dos estudos em fonologia clínica. In: LAMPRECHT, R. R. (Org.). Aquisição da linguagem: questões e análises. Porto Alegre: PUCRS, 1999. p.65-80. 
LAMPRECHT, R. R. et al. Aquisição fonológica do Português: perfil de desenvolvimento e subsídio para terapia. Porto Alegre: ARTMED, 2004.

LARGO, R. et al. Language development of term and preterm children during the first five years of life. Development Medicine \& Child Neurology, Cambridge, n.28, p.333-350, 1986.

\section{LAZZAROTTO-VOLCÃO, C. Modelo padrão de aquisição de contrastes:} uma proposta de avaliação e classificação dos desvios fonológicos. 2009. $217 f$. Dissertação (Doutoramento em Linguística) - Universidade Católica de Pelotas, Pelotas, 2009.

LEVELT, C. On the acquisition of place. The Hague: HAG, 1994. (HIL dissertations in Linguistics, v.8).

LLEÓ, C.; PRINZ, M. Syllable structure parameters and the acquisition of affricates. In: HANNAHS, S. J.; YOUNG-SCHOLTEN, M. (Org.). Focus on phonological acquisition: language acquisition and language disorders. Amsterdam: John Benjamins, 1997. p.143-163.

LUOMA, L. et al. Speech an language development of children born at $\leq 32$ weeks' gestation: a 5-year prospective follow-up study. Development Medicine \& Child Neurology, Cambridge, n.40, p.380-387, 1998.

MACKEN, M. Phonological acquisition. In: GOLDSMITH, J. (Ed.). The handbook of phonological theory. Cambridge: Blackwell, 1995. p.671-696.

MATEUS, M. H.; ANDRADE, E. The phonology of Portuguese. Oxford: Oxford University Press, 2000.

MATZENAUER, C. A aquisição de segmentos do português e o pé métrico. Letras de Hoje, Porto Alegre, v.36, n.3, p.85-99, 2001.

Análise de desvios fonológicos através dos traços distintivos.

1988. 318f. Dissertação (Mestrado em Linguística) - Pontifícia Universidade Católica do Rio Grande do Sul, Porto Alegre, 1988.

MCCARTHY, J.; PRINCE,A. Prosodic morphology. Massachussetts: University of Massachusetts, 1986.

MENN, L.; STOEL-GAMMON, C. Phonological development. In: FLETCHER, P.; MACWHINNEY, B. (Ed.). The handbook of child language. USA: Blackwell, 1995. p.335-350.

MIKKOLA, K. et al. Neurodevelopment outcome at 5 years of age of a national cohort of extremely low birth weight infants who were born in 1996-1997. Pediatrics, Illinois, v.116, n.6, p.1391-1400, 2005. 
MONTGOMERY, G. et al. Effects of prematury on the language development of Hispanic infants. In: ROSSETTI, L. M.; KILE, J. E. Early intervention for special populations of infants and toddlers. Londres: Singular Publishing Group, Inc., 1997. p.251-263.

MORGAN, J.; DEMUTH, K. Signal to syntax: bootstrapping from speech to grammar in early acquisition. Mahwah: LEA Publishers, 1996.

MOTA, H. B. Terapia fonoaudiológica para os desvios fonológicos. Rio de Janeiro: RevinteR, 2001.

NESPOR, M.; VOGEL, I. Prosodic phonology. Dordrecht: Foris, 1986.

NOGUEIRA, P. Desenvolvimento fonológico em crianças dos 3 anos e 6 meses aos 4 anos e 6 meses de idade nascidas com baixo peso. 2007. $180 \mathrm{f}$. Dissertação (Mestrado em Ciências da Fala) - Universidade Católica Portuguesa, Escola Superior de Saúde do Alcoitão, Lisboa, 2007.

OLIVEIRA, C. C. et al. Cronologia da aquisição dos segmentos e das estruturas silábicas. In: LAMPRECHT, R. R. (Org.). Aquisição fonológica do português: perfil de desenvolvimento e subsídios para terapia. Porto Alegre:ARTMED, 2004. p.167-176.

PEIJEN, S. et al. Myelination progression in language-correlated regions in brain of normal children determined by quantitative MRI assessment. International Journal of Pediatric Otorhinolaryngology,Amsterdã, n.72, p.1751-1763, 2008.

PEIXOTO, J. C. et al. Nascer prematuro em Portugal: estudo multicêntrico nacional 1996-2000. Porto: Fundação Bial, 2002.

ROSE, Y. Headedness and prosodic licensing in the L1 acquisition of phonology. 2000. 289f. Tese (Doutorado em Linguística) - McGill University, Montréal, 2000.

RUGOLO, L. M. Follow-up do recém-nascido de muito baixo peso. In: COSTA, H. P.; MARBA, S. T. O recém-nascido de muito baixo peso. São Paulo: Atheneu, 2003. p.469-477.

SAJANIEMI, N. et al. Cognitive development, temperament and behavior at 2 years as indicative of language development at 4 years in pre-term infants. Child Psychiatry and Human Development, New York, v.31, n.4, p.329-346, 2001.

SANTOS, R. Aquisição do acento primário em português brasileiro. 2001. 316f. Dissertação (Doutoramento em Linguística) - Universidade Estadual de Campinas, Campinas, 2001. 
SCARPA, E. Sons preenchedores e guardadores de lugar: relações entre fatos sintáticos e prosódicos na aquisição da linguagem. In: SCARPA, E. Estudos de prosódia. São Paulo: Ed. da UNICAMP, 1999. p.253-284.

SMITH, A. B. Articulation and phonology resource guide for school-age children and adults. Manhattan: Thomson Delmar Learning, 2004.

VIHMAN, M. M. Phonological development: the origins of language in the child. Cambridge: Blackwell, 1996.

VOHR, B. R.; MSALL, M. E. Follow-up of high-risk infants. In: VERGARA, E. R.; BIGSBY, R. Developmental and therapeutic interventions in the NICU. Baltimore: Paul H. Brookes, 2004. p.267-292.

VOIGT, B. et al. Cognitive development in very vs. moderately to late preterm and full-term children: can effortful control account for group differences in toddlerhood? Early Human Development, Amsterdam, n.88, p.307-313, 2012.

WEISSENBORN, J.; HOHLE, B. Approaches to bootstrapping: phonological, lexical, syntactic and neurophysiological aspects of early language acquisition. Amsterdam: John Benjamins, 2001.

YAVAS, M.; HERNANDORENA, C. L.; LAMPRECHT, R. R. Avaliação fonológica da criança: reeducação e terapia. Porto Alegre: ARTMED, 2002.

Avaliação fonológica da criança. Porto Alegre: ARTMED, 1991.

ZOMIGNANI, A.; ZAMBELLI, H.; ANTONIO, A. Desenvolvimento cerebral em recém-nascidos prematuros. Revista Paulista de Pediatria, São Paulo, v.27, n.2, p. 198-203, 2009.

Recebido em junho de 2013.

Aprovado em setembro de 2013. 